\title{
Brane Worlds in Collision
}

\author{
G.W. Gibbons ${ }^{1}$ H. Lü, ${ }^{2}$ C.N. Pope ${ }^{2 *}$ \\ ${ }^{1}$ DAMTP, Centre for Mathematical Sciences, Cambridge University Wilberforce Road, Cambridge CB3 OWA, UK \\ ${ }^{2}$ George P. $\&$ Cynthia W. Mitchell Institute for Fundamental Physics, \\ Texas AEM University, College Station, TX 77843, USA
}

(Dated: January 14, 2005)

\begin{abstract}
We obtain an exact solution of the supergravity equations of motion in which the four-dimensional observed universe is one of a number of colliding D3-branes in a Calabi-Yau background. The collision results in the ten-dimensional spacetime splitting into disconnected regions, bounded by curvature singularities. However, near the D3-branes the metric remains static during and after the collision. We also obtain a general class of solutions representing $p$-brane collisions in arbitrary dimensions, including one in which the universe ends with the mutual annihilation of a positivetension and negative-tension 3-brane.
\end{abstract}

PACS numbers: 11.25.-w, 98.80.Jk, 04.50.+h

The idea that our spacetime is a brane in a higherdimensional world leads naturally to the suggestion that the big bang is the result of a brane collision [1, 2]. Colliding brane-world scenarios offer an alternative to conventional four-dimensional cosmological models, and an important challenge is to make predictions that distinguish between intrinsically higher-dimensional effects and conventional lower-dimensional physics. In order to do so, a better understanding of brane-brane interactions is needed. Studies of D3-D3 and D3-D̄3 collisions have so far been restricted to approximations based on effective field theories on the brane, or on perturbative string-theory techniques 3 , 4]. There have been no exact treatments based on the supergravity equations of motion. The purpose of this letter is to provide an exact treatment in the case of colliding D3-branes, moving in a Calabi-Yau background. Our technique extends to more general brane-collision processes, which we shall also discuss briefly. It requires an extension to $p$-branes of an analysis of black-hole collisions developed in [5, 6], which made use of a remarkable time-dependent generalisation [5] of the static Majumdar-Papapetrou metrics [7]. The collision of extreme Reissner-Nordström black holes had been discussed previously using a modulus-space description [8, 9]. The importance of the work of [6] was that at the expense of introducing a cosmological term, the dynamics could be studied exactly.

The Kastor-Traschen solution [5] of Einstein-Maxwell theory with cosmological constant $\Lambda>0$ takes the form

$$
\begin{aligned}
d s_{4}^{2} & =-H^{-2} d t^{2}+H^{2} d \mathbf{y}^{2}, \quad A=H^{-1} d t, \\
H & =h t+\Phi(\mathbf{y}), \quad \Phi(\mathbf{y}) \equiv \sum_{k} \frac{M_{k}}{\left|\mathbf{y}-\mathbf{y}_{k}\right|},
\end{aligned}
$$

where $h=-\sqrt{\Lambda / 3}$. Note that if the masses $M_{k}$ are set to zero, the solution is de Sitter spacetime, and the apparent singularity at $t=0$ is a mere coordinate artefact. (It corresponds to a null hypersurface separating the maximally-extended de Sitter spacetime into two antipodally-related parts, with $t>0$ and $t<0$. In fact, the involution $t \rightarrow-t$ is the antipodal map.)

When $t$ is negative, the surfaces $t=$ constant are everywhere spacelike. Near each $\mathbf{y}_{k}$, there is an infinite throat which is very similar to the throat of the asymptotically-flat extremal Reissner-Nordström solution, for which $H=1+\Phi(\mathbf{y})$. Far from the throats, however, there is a contracting $k=0$ de Sitter universe $d s_{4}^{2} \sim-d \tau^{2}+a^{2}(\tau) d \mathbf{y}^{2}$, with $a(\tau)=e^{-h \tau}=h t$, and $\tau$ going to $+\infty$.

When $t$ is positive, however, one cannot go too far away from the throats before the function $H$ becomes negative. In fact, the time-dependent locus $H=0$ is a spacetime singularity. In [6], a detailed discussion is given of the locations of the horizons that form around the black holes. The simplest case to consider would be that of two black holes, of masses $M_{1}$ and $M_{2}$, with $M=$ $M_{1}+M_{2}<\frac{1}{4}|h|^{-1}$. This represents the head-on collision of two black holes. As $t$ approaches zero, one has two outer apparent horizons which ultimately coalesce, and a single time-independent Reissner-Nordström-de Sitter black hole is formed at late times $[\underline{6}$.

After the appearance of [5], Maki and Shiraishi obtained a general class of time-dependent multi-particle charged solutions [10], coupled to a scalar field $\phi$. In general, the scalar field couples to the cosmological constant in Liouville-like fashion. Some four-dimensional examples were analysed in [11]. In some cases the cosmological constant can be set to zero. The modulus-space metrics for these multi-particle solutions were given in [12]. The slow motion of NS-NS 5-branes was discussed in [13]. In [14], a particular case was used to study an expanding gas of D0-branes, allowing a comparison of the modulusspace approximation with the exact solution of [10].

In order to discuss brane scattering, we shall lift some of the Maki-Shiraishi solutions to higher dimensions. Their Lagrangian in $(N+1)$ dimensions is

$$
\mathcal{L}=R-\frac{4}{N-1}(\partial \phi)^{2}-e^{-4 a \phi /(N-1)} F^{2}-\Lambda e^{4 b \phi /(N-1)} .
$$


We restrict attention to solutions described by Case (II) in [10], for which

$$
\begin{aligned}
& d s^{2}=-V^{-\frac{2(N-2)}{N-2+a^{2}}} d t^{2}+a^{2}(t) V^{\frac{2}{N-2+a^{2}}} d \mathbf{y}^{2}, \\
& A=-\sqrt{\frac{N-1}{2\left(N-2+a^{2}\right)}} \frac{1}{\sqrt{C}(a(t))^{a^{2}}} V^{-1} d t, \\
& e^{-4 a \phi /(N-1)}=C(a(t))^{2 a^{2}} V^{2 a^{2} /\left(N-2+a^{2}\right)},
\end{aligned}
$$

where

$$
\begin{aligned}
V & =1+\sum_{k} \frac{\mu_{k}}{\sqrt{C}(a(t))^{N-2+a^{2}}(N-2)\left|\mathbf{y}-\mathbf{y}_{k}\right|^{N-2}}, \\
a(t) & =\left(\frac{t}{t_{0}}\right)^{1 / a^{2}}, \quad \frac{N}{a^{2}}-1=\frac{a^{2} t_{0}^{2} \Lambda}{C(N-1)} .
\end{aligned}
$$

By contrast with the Kastor-Traschen case, the presence of the scalar field allows us to take $\Lambda=0$, by choosing $a^{2}=N$. In the notation of [15], this coupling strength of the dilaton to the Maxwell field is characterised by the constant $\Delta$ taking the value 4 . In fact $\Delta=4$ is precisely the coupling that one encounters, in any dimension, for a Maxwell field arising from the Kaluza-Klein vector in a circle reduction [15]. It is also the coupling strength for any field strength in maximal supergravity in any dimension [15, 16]. Thus, for example, if $N=9$ the vector field is the Ramond-Ramond vector of type IIA string theory, which may also be interpreted as arising from M-theory via Kaluza-Klein reduction [14].

To construct colliding D3-branes we require $N=6$, and the Maxwell field strength may be viewed as arising from the 5 -form of type IIB supergravity. Lifting the solution from seven to ten dimensions, we obtain a time-dependent D3-brane solution, in which the only non-trivial fields are the metric and the 5 -form, given by

$$
\begin{aligned}
d s_{10}^{2} & =H^{-1 / 2}\left(-d t^{2}+d \mathbf{x}^{2}\right)+H^{1 / 2} d \mathbf{y}^{2}, \\
F_{(5)} & =d t \wedge d^{3} \mathbf{x} \wedge d H^{-1}+*\left(d t \wedge d^{3} \mathbf{x} \wedge d H^{-1}\right), \\
H & =h t+\Phi(\mathbf{y}), \quad \Phi(\mathbf{y}) \equiv \sum_{k} \frac{M_{k}}{\left|\mathbf{y}-\mathbf{y}_{k}\right|^{4}}
\end{aligned}
$$

where $h$ is an arbitrary constant (which we shall take to be negative, to parallel the discussion in [6]). Sending $t \rightarrow t+1 / h$ one gets a standard supersymmetric static D3-brane if $h=0$. However, the time-dependent $h \neq 0$ solution is non-supersymmetric.

If the non-negative constants $M_{k}$, which correspond to the 3-brane charges, all vanish, then we obtain a vacuum solution of the Einstein equations, which is a generalisation of the familiar Kasner solution,

$$
\begin{aligned}
d s_{10}^{2} & =(h t)^{-1 / 2}\left(-d t^{2}+d \mathbf{x}^{2}\right)+(h t)^{1 / 2} d \mathbf{y}^{2} \\
& =-d \tau^{2}+\left(\frac{3 h \tau}{4}\right)^{-2 / 3} d \mathbf{x}^{2}+\left(\frac{3 h \tau}{4}\right)^{2 / 3} d \mathbf{y}^{2},
\end{aligned}
$$

where we have defined $h \tau=\frac{4}{3}(h t)^{3 / 4}$. The singularity at $t=0$ is, by contrast with the de Sitter case, a true curva- ture singularity, and the Kasner metric cannot be continued from negative to positive $t$. In the ten-dimensional metric the three spatial dimensions are increasing in size as $t$ increases towards zero from an initial negative value. From the point of view of four dimensions, the interpretation is simplest in the Einstein conformal gauge, which is achieved by writing

$$
d s_{10}^{2}=e^{2 \alpha \varphi} d s_{4}^{2}+e^{-2 \alpha \varphi / 3} d \mathbf{y}^{2},
$$

where $\alpha^{2}=3 / 16$. Introducing the Einstein proper-time coordinate $T=3 h \tau^{2} / 8$, the four-dimensional Einsteinframe metric becomes

$$
d s_{4}^{2}=-d T^{2}+\left(\frac{3}{2} h T\right)^{2 / 3} d \mathbf{x}^{2} .
$$

This is of the form expected for gravity coupled to a massless scalar field, which behaves like a perfect fluid with a stiff-matter equation of state for which the energy density equals the pressure. In the Einstein conformal gauge, the spatial 3-sections contract as $T$ increases towards zero from an initial negative value; $T=0$ is a big-crunch singularity. If instead we run time backwards, the solution represents an expanding universe with a big-bang singularity. The discussion above emphasises the point that four-dimensional physics should be analysed in the Einstein conformal frame.

Now let us consider the case where the constants $M_{k}$ are non-zero. Starting with $t$ negative but increasing towards zero, the solution represents 3 -branes moving in a background Kasner universe, in which, measured in the ten-dimensional metric, the transverse space contracts while the D3-brane world volume expands. From the point of view of four dimensions, the situation is similar to the pure Kasner case discussed above, as long as one stays well away from all of the D3-branes, because the relevant conformal factor is

$$
e^{-2 \alpha \varphi}=(h t)^{3 / 2}\left(1+\frac{1}{h t} \sum_{k} \frac{M_{k}}{\left|\mathbf{y}-\mathbf{y}_{k}\right|^{4}}\right)^{3 / 2} .
$$

At any fixed negative $t$ the surfaces of constant time are smooth, asymptotically flat and non-compact. Near each 3-brane, $\mathbf{y} \sim \mathbf{y}_{k}$, the 10-metric is static, and wellapproximated by the product metric on $\mathrm{AdS}_{5} \times S^{5}$. Because the transverse directions are inhomogeneous, the four-dimensional interpretation depends upon where one is in the transverse space.

The situation as $t$ goes to zero is more complicated than in the pure Kasner case. If $t$ is exactly zero, we obtain a non-singular ten-dimensional configuration that is not asymptotically flat, which may be thought of as a union of $\mathrm{AdS}_{5} \times S^{5}$ components. An analogous solution has been discussed for $\mathrm{AdS}_{2} \times S^{2}$ in [17, 18, 19], in connection with anti-de Sitter fragmentation.

If the charges $M_{k}$ are non-zero the solution continues to exist in a neighbourhood of each 3 -brane when $t$ 


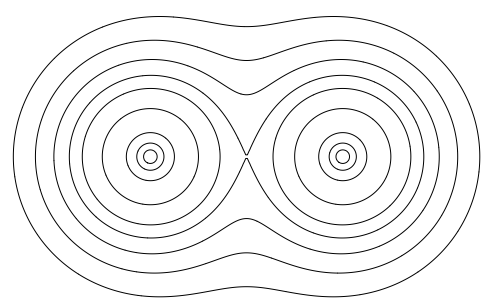

FIG. 1: The level sets of $\Phi(\mathbf{y})$ for two mass points: The universe after the collision is confined inside a level set $\Phi(\mathbf{y})>$ $-h t$. As time progresses, the level set $\partial D_{t}$ shrinks and splits into two components, which then shrink around the two D3branes.

becomes positive, by contrast with the Kasner solution. Specifically, the metric is

$$
\begin{aligned}
d s_{10}^{2}= & (-h t)^{-1 / 2}\left(\frac{\Phi(\mathbf{y})}{(-h t)}-1\right)^{-1 / 2}\left(-d t^{2}+d \mathbf{x}^{2}\right) \\
& +(-h t)^{1 / 2}\left(\frac{\Phi(\mathbf{y})}{(-h t)}-1\right)^{1 / 2} d \mathbf{y}^{2}
\end{aligned}
$$

where $\Phi(\mathbf{y})$ is given in (5). For all positive times, the metric exists inside a domain $D_{t}$ bounded by the level set $\Phi(\mathbf{y})=-h t$. Inside the domain $D_{t}$, but away from the D3-branes themselves, the transverse dimensions expand while the three spatial dimensions contract even with respect to the four-dimensional Einstein-frame metric, if one stays at fixed $\mathbf{y}$. However, if one moves in the transverse space in such a way that $\Phi(\mathbf{y}) /(-h t)$ remains approximately constant (and greater than 1), then the corresponding four-dimensional universe has expanding spatial sections.

At small positive $t$, the domain $D_{t}$ is a large connected ball containing all the D3-branes. As $t$ increases, the domain $D_{t}$ shrinks, and eventually splits into disconnected pieces which shrink down onto each mass point. In other words, the universe splits into disconnected regions containing the individual D3-branes. Near each D3-brane, however, the metric is static, and again well approximated by the product metric on $\mathrm{AdS}_{5} \times S^{5}$.

Consider, for example, the case of two mass points, which is illustrated in Fig. 1. At negative times the D3branes are approaching one another in a head-on collision, with the universe contracting about them. The D3branes never actually meet, but rather scatter off each other into a universe bounded by spacetime singularities.

In the solutions we have discussed so far, the sixdimensional transverse space is flat. In more realistic examples one might want to consider D3-branes moving in a Calabi-Yau manifold. In fact, the solutions given by (5D) may be generalised to the case where the flat transverse metric $d \mathbf{y}^{2}$ is replaced by any Ricci-flat 6 -metric, and $H=h t+\Phi(\mathbf{y})$ where $\Phi(\mathbf{y})$ is any harmonic function on the Ricci-flat 6-manifold. In particular, we can take the Ricci-flat manifold to be Calabi-Yau.
The time-dependent D3-brane solutions that we obtained above admit a straightforward generalisation to the case of an arbitrary $p$-brane supported by a $(p+2)$ form field strength with a dilaton coupling characterised by $\Delta=4$ in the notation of [15]. The relevant $D$ dimensional Lagrangian can be expressed as

$$
\mathcal{L}=R-\frac{1}{2}(\partial \phi)^{2}-\frac{1}{2(p+2) !} e^{c \phi} F_{(p+2)}^{2},
$$

where

$c^{2} \equiv \Delta-\frac{2(p+1)(D-p-3)}{D-2}=4-\frac{2(p+1)(D-p-3)}{D-2}$.

The time-dependent $p$-brane solution is given by

$$
\begin{aligned}
& d s^{2}=H^{-\frac{D-p-3}{D-2}}\left(-d t^{2}+d \mathbf{x}^{2}\right)+H^{\frac{p+1}{D-2}} g_{m n}(y) d y^{m} d y^{n}, \\
& F_{(p+2)}=d t \wedge d^{p} \mathbf{x} \wedge d H^{-1} \\
& \phi=\frac{1}{2} c \log H, \quad H=h t+\Phi(y)
\end{aligned}
$$

and $h$ is an arbitrary constant. Here $g_{m n}(y) d y^{m} d y^{n}$ is any Ricci-flat $(D-p-1)$-metric, and $\Phi(y)$ is an harmonic function in this metric. One might, for example, take the flat metric $g_{m n}(y) d y^{m} d y^{n}=d \mathbf{y}^{2}$ and $\Phi(\mathbf{y})=\sum_{k} M_{k} /\left|\mathbf{y}-\mathbf{y}_{k}\right|^{D-p-3}$.

These solutions can be derived by lifting the Case (II) time-dependent Maki-Shiraishi solutions [10], setting $a^{2}=N$ so that $\Delta=4$. Alternatively, they can be derived by using T-duality symmetries to relate them to pp-waves. We begin by noting that the pp-wave metric

$d s^{2}=2 d x^{+} d x^{-}+\frac{4}{\left(2 h x^{+}\right)^{2}} \Phi(y)\left(d x^{+}\right)^{2}+g_{m n}(y) d y^{m} d y^{n}$

is Ricci-flat for any $\Phi(y)$ that is harmonic in the Ricciflat metric $g_{m n}(y) d y^{m} d y^{n}$. It is invariant under the boost given by $x^{+} \rightarrow \lambda x^{+}, x^{-} \rightarrow \lambda^{-1} x^{-}$. We may therefore perform a Kaluza-Klein reduction on this boost symmetry by first introducing coordinates $(t, z)$, given by $x^{-}=t e^{\frac{1}{2} h z}$ and $x^{+}=-(2 / h) e^{-\frac{1}{2} h z}$. This gives

$$
d s^{2}=-H^{-1} d t^{2}+H\left(d z+H^{-1} d t\right)^{2}+g_{m n}(y) d y^{m} d y^{n},
$$

with $H=h t+\Phi(y)$. Reducing on the boost Killing vector $\partial / \partial z$ gives a time-dependent 0 -brane. If one is starting from $D=11$ supergravity, this gives the D0brane that was discussed in [14], which is the special case $N=9=a^{2}$ of Maki and Shiraishi.

By performing a sequence of T-duality transformations in the standard way (see, for example, [20]), this immediately gives us all the time-dependent $p$-brane solutions that we have presented above. A further extension is to consider harmonic intersecting $p$-branes. It is straightforward to see that any one (but only one) of the harmonic functions can be generalised from $1+\Phi(y)$ to $h t+\Phi(y)$.

A further generalisation is to apply a discrete electric/magnetic duality transformation in four dimensions. 
Consider, for example, the metric representing a maximally electrically charged Kaluza-Klein black hole:

$$
\begin{aligned}
d s_{4}^{2} & =-H^{-1 / 2} d t^{2}+H^{1 / 2} d \mathbf{y}^{2}, \\
F & =-d t \wedge d H^{-1}, \quad \phi=\frac{1}{2} \sqrt{3} \log H,
\end{aligned}
$$

with $H=h t+\Phi(\mathbf{y})$. After making the duality transformation we obtain the magnetic solution, with the same metric and with

$$
F=d A=*_{3} d \Phi, \quad \phi=-\frac{1}{2} \sqrt{3} \log H
$$

where $*_{3}$ denotes the Hodge dual in the flat 3 -metric $d \mathbf{y}^{2}$. Lifting to five dimensions, we obtain the Ricci-flat metric

$$
d s_{5}^{2}=-d t^{2}+H d \mathbf{y}^{2}+H^{-1}(d z+A)^{2} .
$$

The last two terms in (18) give a time-dependent sequence of hyper-Kähler 4-metrics. The interpretation of the metric (16) is that it represents an assembly of freelyexpanding point particles in a background Friedman universe with a scale factor $a(T)$ proportional to $T^{1 / 3}$. In the slow-motion approximation the motion is free and hence the branes separate linearly with time [21].

It would be of great interest to study the stability of Hořava-Witten brane models by finding exact timedependent solutions; some preliminary results are reported in [22]. Here, we present a different colliding brane-world model, which however captures the essence of all brane-world instabilities. We take $\Phi(\mathbf{y})=M\left|y_{1}\right|$ in (5), and reduce on $\left(y_{2}, \ldots, y_{6}\right)$, obtaining the 5 -metric

$d s_{5}^{2}=-\left(h t+M\left|y_{1}\right|\right)^{1 / 3}\left(-d t^{2}+d \mathbf{x}^{2}\right)+\left(h t+M\left|y_{1}\right|\right)^{4 / 3} d y_{1}^{2}$,

which describes a negative-tension time-dependent 3brane at $y_{1}=0$, supported by a 5 -form. If the solution is reflected about $y_{1}=L$, and if $y_{1}=2 L$ is identified with $y_{1}=0$, we obtain an $S^{1} / Z_{2}$ orientifold with an additional positive-tension brane at $y_{1}=L$. The proper distance between the 3 -branes is $\left.6\left[(h t+M L)^{5 / 3}-(h t)^{5 / 3}\right)\right] /(5 M)$. This decreases monotonically as $t$ increases to 0 . Defining $T=2(h t+M L)^{3 / 2} /(3 h)$, the Einstein metric induced on the positive-tension brane is of the FriedmanLemaitre form (8), driven by a massless scalar field, i.e. the radion. At $t=0$ a singularity forms on the negativetension brane, and moves towards the positive-tension brane, causing the complete annihilation of the universe at $t=M L /(-h)$, i.e. $T=0$. This is the way the braneworld ends, not with a whimper but a bang.

G.W.G. is grateful to Indrajit Mitra for helpful discussions, and to the George P. \& Cynthia W. Mitchell Institute for Fundamental Physics for hospitality during the course of this work.

* Research is supported in part by DOE grant DE-FG0395ER40917
[1] G.R. Dvali and S.H.H. Tye, Brane inflation, Phys. Lett. B450, 72 (1999), hep-ph/9812483

[2] J. Khoury, B.A. Ovrut, P.J. Steinhardt and N. Turok, The ekpyrotic universe: Colliding branes and the origin of the hot big bang, Phys. Rev. D64, 123522 (2001), hep-th/0103239

[3] N. Turok, M.J. Perry and P.J. Steinhardt, $M$ theory model of a big crunch / big bang transition, Phys. Rev. D70, 106004 (2004), hep-th/0408083

[4] L. McAllister and I. Mitra, Relativistic D-brane scattering is extremely inelastic, hep-th/0408085.

[5] D. Kastor and J.H. Traschen, Cosmological multiblack-hole solutions, Phys. Rev. D47, 5370 (1993), hep-th/9212035.

[6] D.R. Brill, G.T. Horowitz, D. Kastor and J.H. Traschen, Testing cosmic censorship with black hole collisions, Phys. Rev. D49, 840 (1994), gr-qc/9307014.

[7] S.D. Majumdar, Phys. Rev. 72, 930, (1947); A. Papapetrou, Proc. Roy. Irish. Ac. Vol. 851, (191), (1947).

[8] G.W. Gibbons and P.J. Ruback, The motion of extreme Reissner-Nordstrom black holes in the low velocity limit, Phys. Rev. Lett. 57, 1492 (1986).

[9] R.C. Ferrell and D.M. Eardley, Slow motion scattering and coalescence of maximally charged black holes, Phys. Rev. Lett. 59 (1987) 1617.

[10] T. Maki and K. Shiraishi, Multi-black hole solutions in cosmological Einstein-Maxwell dilaton theory, Class. Quant. Grav. 10, 2171 (1993).

[11] J.H.Horne and G.T.Horowitz, Cosmic censorship and the dilaton, Phys. Rev. D48, 5457 (1993), hep-th/9307177.

[12] K. Shiraishi, Moduli space metric for maximally charged dilaton black holes, Nucl. Phys. B402, 399 (1993).

[13] A.G. Felce and T.M. Samols, Low-energy dynamics of string solitons, Phys. Lett. B308, 30 (1993), hep-th/9211118

[14] D.Z. Freedman, G.W. Gibbons and M. Schnabl, Matrix cosmology, in "The New Cosmology", R.E. Allen, D.V. Nanopoulos, C.N. Pope, AIP conference proceedings, Vol. 743, 286, (2004), hep-th/0411119,

[15] H. Lü, C.N. Pope, E. Sezgin and K.S. Stelle, Stainless super p-branes, Nucl. Phys. B456, 669 (1995), hep-th/9508042.

[16] M.J. Duff, R.R. Khuri and J.X. Lu, String solitons, Phys. Rept. 259, 213 (1995), hep-th/9412184

[17] D.R. Brill, Splitting of an extremal Reissner-Nordstrom throat via quantum tunneling, Phys. Rev. D46, 1560 (1992), hep-th/9202037.

[18] J.M. Maldacena, J. Michelson and A. Strominger, Anti-de Sitter fragmentation, JHEP 9902, 011 (1999), hep-th/9812073.

[19] S. Ng and M.J. Perry, Brane splitting via quantum tunneling, Nucl. Phys. B634, 209 (2002), hep-th/0106008.

[20] E. Bergshoeff, C.M. Hull and T. Ortin, Duality in the type II superstring effective action, Nucl. Phys. B451, 547 (1995), hep-th/9504081.

[21] P.J. Ruback, The motion of Kaluza-Klein monopoles, Commun. Math. Phys. 107, 93 (1986).

[22] W. Chen, Z.W. Chong, G.W. Gibbons, H. Lü and C.N. Pope, Horava-Witten stability: Eppur si muove, hep-th/0502077 